\title{
Material design of nanocellulose/polymer composites via Pickering emulsion templating
}

\author{
Shuji Fujisawa (iD ${ }^{1}$
}

Received: 17 June 2020 / Revised: 7 August 2020 / Accepted: 20 August 2020 / Published online: 28 September 2020

(c) The Author(s) 2020. This article is published with open access

\begin{abstract}
Cellulose nanofiber (CNF) is a crystalline fiber composed of a bundle of cellulose molecular chains and is expected to be used as a new biomass-derived nanomaterial. The CNF has a unique morphology: a few to tens of nanometer width and a submicrometer to micrometer length. Its application to various materials, in particular its utilization as a polymer reinforcing material, has been anticipated due to its excellent mechanical properties. However, CNFs and plastics are generally hard to mix, and thus, it is difficult to combine them at the nanolevel. In this review, we describe the CNF/polymer nanocompositing process from Pickering emulsion. We use $\sim 3 \mathrm{~nm}$-wide wood-derived CNFs and report on the preparation of CNF/polymer homogenous composite films. We also introduce a new type of $\mathrm{CNF} /$ polymer composite, a core-shell microparticle, using this Pickering emulsion as a template.
\end{abstract}

\section{Introduction}

Plastics are used for multiple purposes as parts of automobiles, home electrical appliances, food packaging materials, and medical equipment and thus constitute essential materials in our lives. Reinforcing materials are often added to these plastics to improve their mechanical properties. Common reinforcing materials include glass and carbon fibers; hence, composite materials acquire the strength and thermal dimensional stability that plastics cannot attain on their own. However, as the energy demand for the production and discarding of these reinforcing materials is high, plastic reinforcement using environmentally friendly materials has become a priority toward the implementation of a low-carbon society.

In recent years, cellulose nanofibers (CNFs) have been used as a natural carbon-neutral reinforcing material. In particular, wood-derived CNFs have a characteristic shape and are $\sim 3 \mathrm{~nm}$ in width and several micrometers in length. They have been demonstrated to have excellent mechanical

Shuji Fujisawa

afujisawa@mail.ecc.u-tokyo.ac.jp

1 Department of Biomaterials Sciences, Graduate School of Agricultural and Life Sciences, The University of Tokyo, Tokyo 113-8657, Japan and thermomechanical properties, with high strength (2-6 GPa) [1, 2], high elastic moduli (130-150 GPa) [3-5], and low thermal expansion coefficients $\left(4-6 \mathrm{ppm} \mathrm{K}^{-1}\right)[6,7]$. Taking advantage of these characteristics of CNFs, ongoing research has been extensively focused on utilizing them as a reinforcing material for plastics since the mid-1990s [8-10]. To fully elicit the reinforcing effect of CNFs, CNFs must be homogenously dispersed in a composite material. However, obtaining a homogenous composition of CNFs and commodity plastics has been difficult due to the high interfacial energy. Therefore, the composition difficulty poses the biggest challenge for the production of such materials.

To date, we have sought to develop homogeneously mixed CNF/polymer composites using a CNF prepared through chemical pretreatment termed 2,2,6,6-tetramethylpiperidinyl-1-oxyl (TEMPO)-mediated oxidation $[11,12]$. TEMPO-mediated oxidation is a chemical reaction that enables selective conversion of primary hydroxy groups into carboxylate groups in water. The oxidation selectively introduces carboxylate groups onto the surface of cellulose microfibrils. The increased osmotic pressure between the fibrils and mild mechanical treatment in water allow the preparation of CNFs with nanosized widths and good colloidal stability in water. Moreover, tailoring the chemical structure of the carboxy groups effectively enables the dispersion of CNFs in various organic solvents [13-15]. Homogeneous CNF/polymer composites can be prepared by drying a mixture of a plastic and CNF dispersions in 

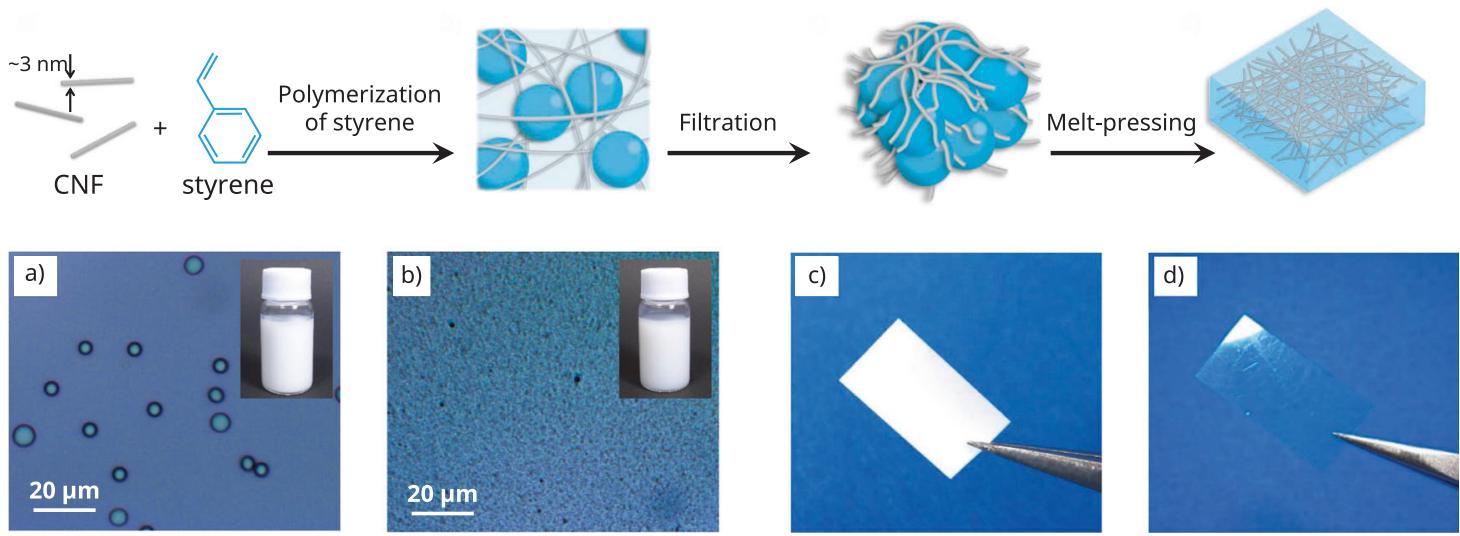

Fig. 1 Schematic illustration of the synthesis of a CNF/PS composite film. a Monomer-in-water emulsion stabilized by CNFs, $\mathbf{b}$ dispersion of $\mathrm{CNFs}$ and PS nanoparticles in water, c CNF/PS nanoparticle

composite sheet, and d CNF/PS composite film. Copyright 2017, American Chemical Society
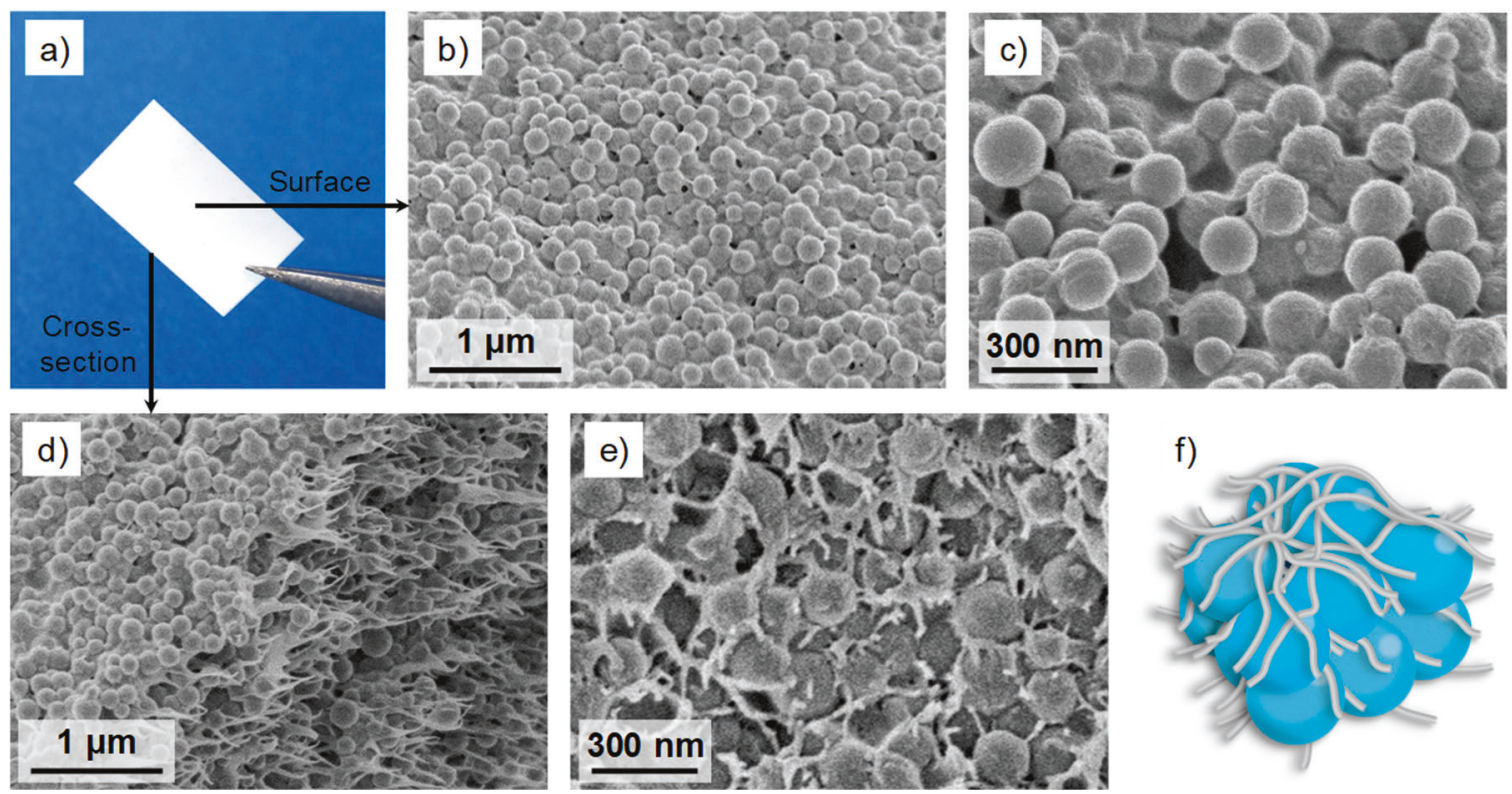

Fig. 2 a Photograph, b-e SEM images, and $\mathbf{f}$ schematic illustration of a PS/CNF composite with 12\% w/w CNFs before melt pressing. b, c Film surface, d edge, and e side surface. Copyright 2017, American Chemical Society

organic solvents [14, 16, 17]. However, such a process is highly time and energy consuming.

In this review, we sought to develop a homogenous composite of CNFs and a hydrophobic plastic using an aqueous solution process, which does not require organic solvents or melt blending procedures. CNFs are known to serve as an excellent emulsifier and form stable Pickering emulsions [18-20]. Pickering emulsions are emulsions stabilized by solid particles. Compared to surfactant molecules, the solid particles more strongly adsorb at liquid/ liquid interfaces. Therefore, Pickering emulsions generally show better stability than emulsions stabilized by surfactants. In the present study, a CNF-stabilized monomer-inwater emulsion was used as a starting point to design a
$\mathrm{CNF} /$ polymer composite structure [21]. Several studies on polymer composite preparation through Pickering emulsions have been reported, where nanocelluloses [22, 23], microfibrillated lignocellulose [24], or chitin nanofibrils [25-27] were used as stabilizers. Furthermore, we report the synthesis of core-shell microparticles using this Pickering emulsion as a template [28].

\section{CNF/polymer nanocomposite film}

In this study, polystyrene (PS) was used as a matrix. First, an aqueous dispersion of CNFs was mixed with styrene monomers, into which an initiator was dissolved. This mixture then underwent ultrasound treatment, which 

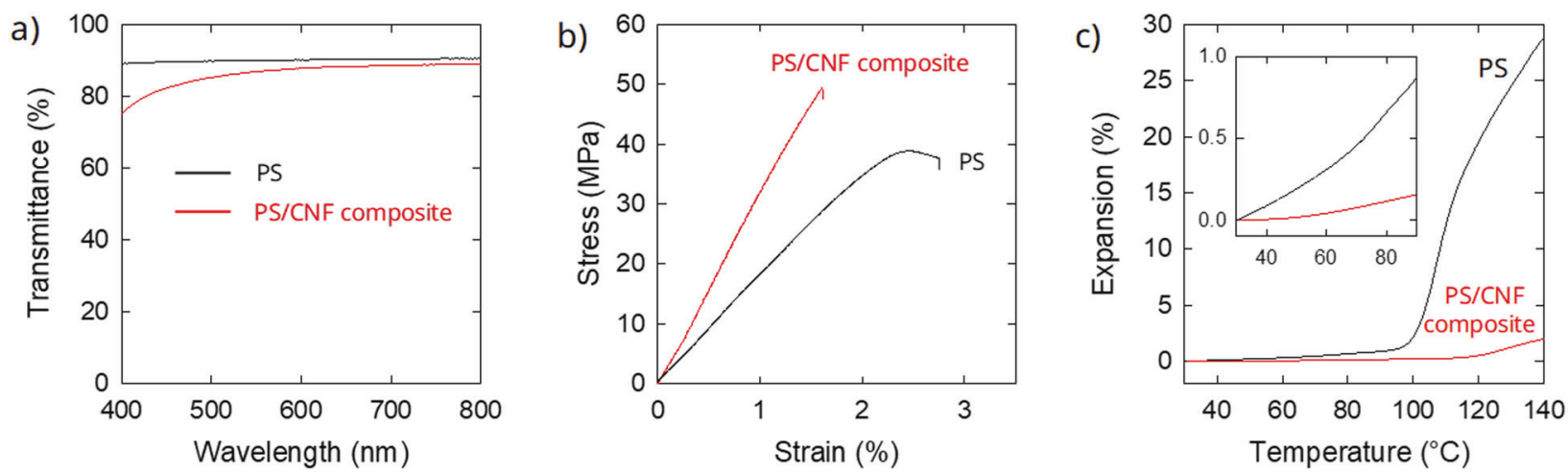

Fig. 3 Properties of a PS/CNF composite film with $12 \% \mathrm{w} / \mathrm{w}$ CNFs after melt pressing. a UV-vis transmittance spectra, b stress-strain curves, and c thermal expansion behavior of the PS/CNF composite and PS films. Copyright 2017, American Chemical Society

resulted in the formation of a CNF-stabilized styrene-inwater emulsion (Fig. 1a). As the interfacial free energy between styrene and water is high, usually, styrene and water ultimately undergo phase separation without being homogenously mixed. In contrast, in the present system, CNFs were adsorbed onto the water/styrene interface, metastabilizing the system. The emulsion using solid particles as a dispersion stabilizer is generally termed a Pickering emulsion. The Pickering emulsion in this work was quite stable, even when it was kept at room temperature for more than a week. The monomer droplets were stably dispersed in water, retaining the size distribution. The repulsive force between the droplets is mainly caused by the osmotic pressure due to the carboxylate groups on the surface of TEMPO-oxidized CNFs.

The obtained emulsion was heated at $70^{\circ} \mathrm{C}$ to polymerize the monomer. As a result, in the polymerization process, the structure of the microemulsion broke down, and a structure in which PS nanoparticles were homogenously dispersed in an aqueous dispersion of CNFs (Fig. 1b) was formed. This polymerization mechanism resembles the method termed dispersion polymerization [29-31], where polymerization occurs in poor solvents. Scanning electron microscopy showed that during the course of polymerization, PS nanoparticles were squeezed out from monomer droplets and deposited in the CNF aqueous dispersion.

Via filtration of the dispersion through membrane filters, CNF/PS nanoparticle composite sheets can be prepared (Fig. 1c). The PS yield was $84 \%$, and the CNF volume fraction within the sheet calculated from the yield was $7 \%$. Observation of the sheets using electron microscopy revealed the formation of composites of CNFs and PS nanoparticles with diameters of $\sim 150 \mathrm{~nm}$ (Fig. 2). The PS nanoparticles were observed to homogeneously form composites with CNFs both on the surfaces and in the interior of the sheets. Importantly, nanocompositing of PS and CNFs was achieved using an aqueous solution process. As noted above, fabricating homogeneous $\mathrm{CNF} /$ polymer composites is time and energy consuming. In contrast, the nanocomposite sheets obtained with the present method can be easily formed by homogenously depositing PS nanoparticles in a CNF network in water, followed by their collection through filtration. Measurement based on a size exclusion chromatography-multiangle laser-light scattering detector revealed that the polymerized PS was composed of straight chains, with an average molecular weight of 1560 000 (polydispersity index of 1.6), indicating an extremely high degree of polymerization.

The resulting CNF/PS nanoparticle sheets had a highly porous structure and therefore low transparency. Nonetheless, they formed transparent CNF/PS composite films following a $30 \mathrm{~s}$ hot press process at $160{ }^{\circ} \mathrm{C}$ (Fig. 1d). After this, the PS nanoparticles formed films due to the glass transition (Fig. 2e, f). The light transmittance rate at $600 \mathrm{~nm}$ was observed to be $88 \%$; composites were formed without serious loss of transparency of the PS film $(90 \%$ at $600 \mathrm{~nm})$ (Fig. 3a). Although a transparent film can be obtained here, a heating process is necessary after the polymerization. In future work, development of a process without heating may be needed for commercial use. Tensile testing of the films demonstrated that the addition of CNFs increased the Young's modulus and strength (Fig. 3b). However, the elongation at break was noted to be reduced with the addition of CNFs. In simple terms, although the material became stiff and strong, it also became quite brittle. We assumed that this was due to the poor interactions at the CNF-PS interface. This is known to be a common property of CNF-based polymer composites, and to reduce their brittleness, the interfacial interactions must be improved through modification of the surface chemical structures of CNFs [14, 17]. Furthermore, the thermal dimensional stability was shown to be improved by the formation of a network of CNFs in the PS matrix (Fig. 3c). This improvement was particularly striking at temperatures greater than $100^{\circ} \mathrm{C}$, which corresponds to the glass 


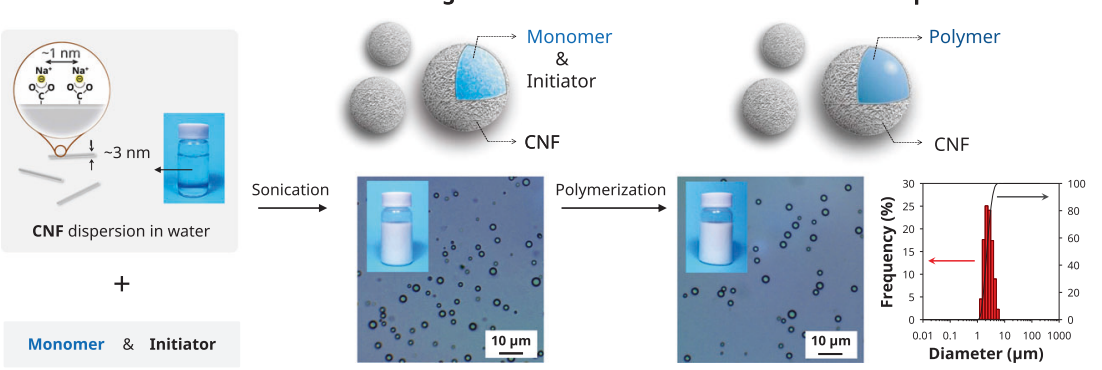

Fig. 4 Schematic illustration of the synthesis of CNF-shelled microparticles at a 10:90 ratio (o/w). CNF-stabilized Pickering monomer-inwater emulsion (center), CNF-shelled microparticle dispersion in water, and size distribution of $\mathrm{CNF} /$ polymer microparticles determined by dynamic light scattering analysis showing a median diameter of 3.0 $\mu \mathrm{m}$ (right). Copyright 2019, The Royal Society of Chemistry
Fig. 5 a Illustration and $\mathbf{b}-\mathbf{d}$ SEM images of CNF-shelled microparticles and surface (bottom right). e Illustration and f, g SEM images of the cross section of microparticles embedded in acrylic resin. Black arrows indicate the CNF shell. Copyright 2019, The Royal Society of Chemistry
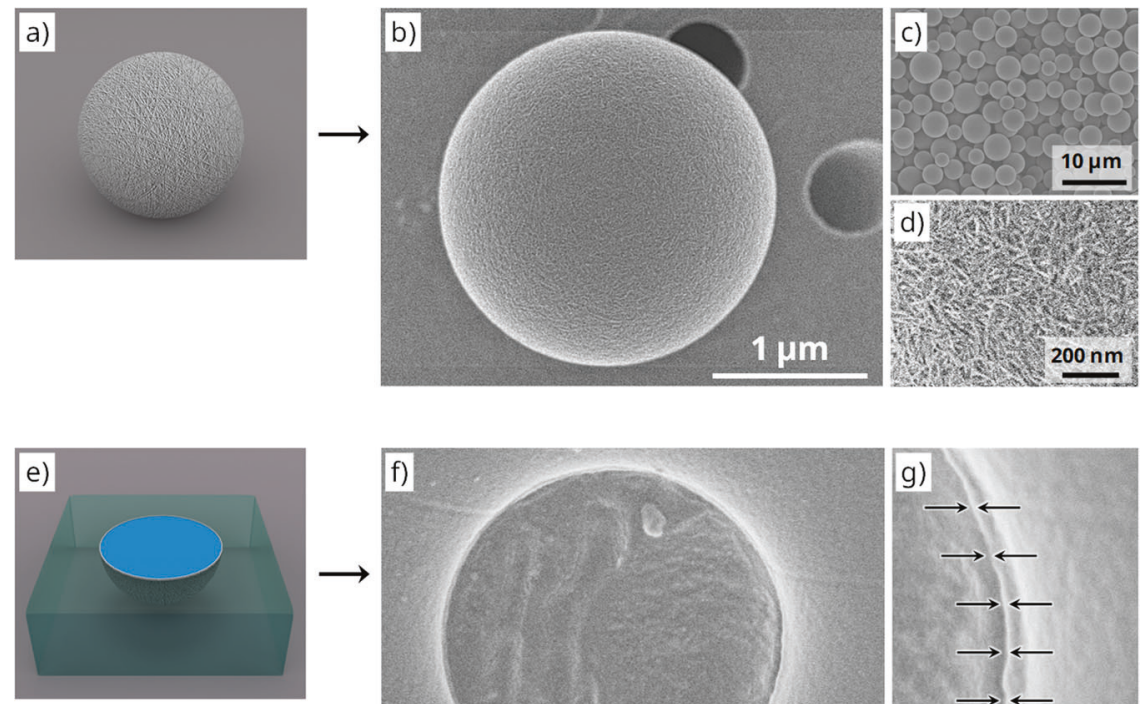
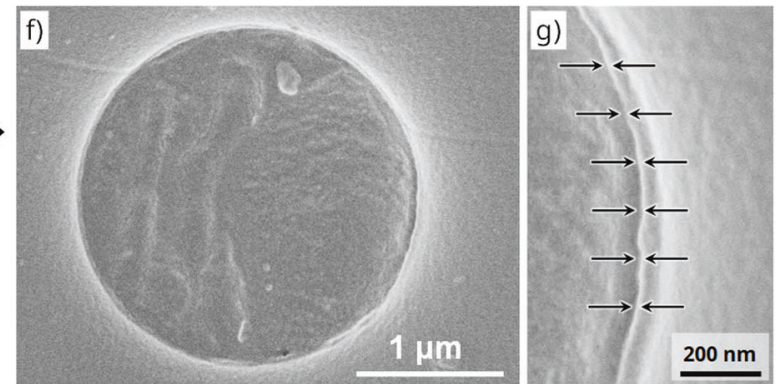

transition region of PS; the disadvantage of the propensity of PS to stretch when heated was successfully compensated by the addition of CNFs. These tensile and thermomechanical properties were shown to be almost comparable to those of CNF/PS composites [16] prepared from organic solvents, indicating that the use of the aqueous solution process in the present study almost achieved the ideal CNF dispersion in PS.

\section{Cellulose nanofiber-shelled polymer microparticles}

Using the aforementioned $\mathrm{CNF} /$ monomer Pickering emulsion and controlling the polymerization conditions, $\mathrm{CNF} /$ polymer microparticles that preserved the droplet shape of the emulsion (Fig. 4) were successfully prepared. Goi et al. reported that the interfacial tension between oil and water plays a significant role in stabilizing CNF-based Pickering emulsions [32]. In preparing $\mathrm{CNF} /$ polymer composites through emulsion templating, the interfacial tension between the monomer liquid and water is also an important factor in controlling the composite structure. In the present study, we used divinylbenzene as a monomer in place of styrene. The average diameter of the obtained microparticles was $\sim 3 \mu \mathrm{m}$, showing that the initial droplet size in the emulsion was maintained. Furthermore, as the surfaces of these microparticles were densely covered with TEMPO-oxidized CNFs, which have carboxylate groups on the surfaces, the zeta potential of the microparticles was shown to be $\sim-50 \mathrm{mV}$. Therefore, the microparticles exhibited good dispersibility in water. Observation of the surfaces of microparticles using scanning electron microscopy revealed that the surfaces were densely covered with the CNFs (Fig. 5b-d). Further observation of cross sections revealed no voids in the interior of the particles, with the interior being uniformly filled with polymers. Based on the scanning electron microscopy results, the thickness of the CNFs on the surface of the particles was estimated to be $\sim 8 \mathrm{~nm}$ (Fig. $5 \mathrm{f}$ 
a)<smiles>CN(C)c1ccc2nc3ccc(N(C)C)cc3[s+]c2c1</smiles>

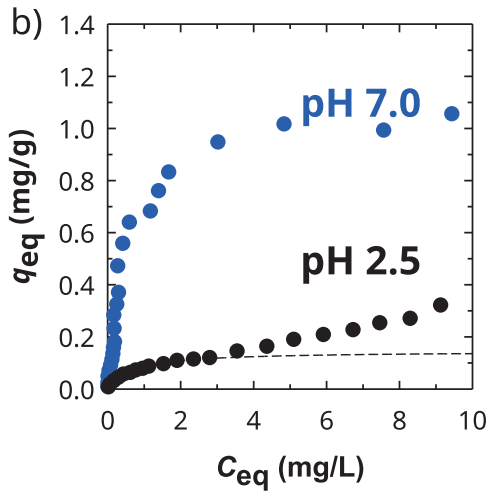

d)
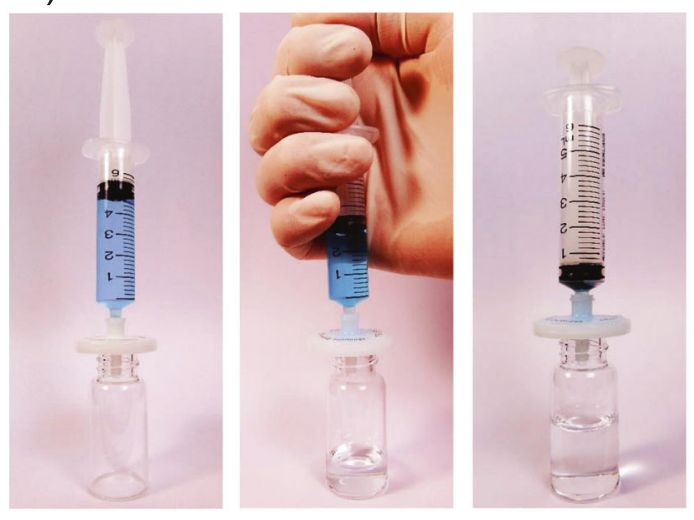

e)
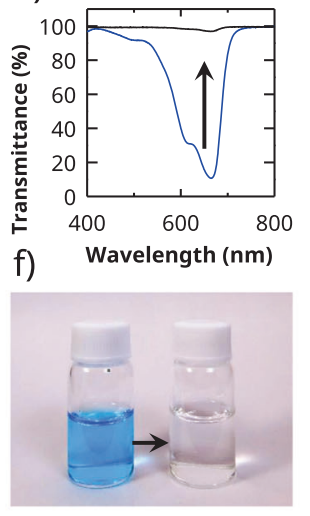

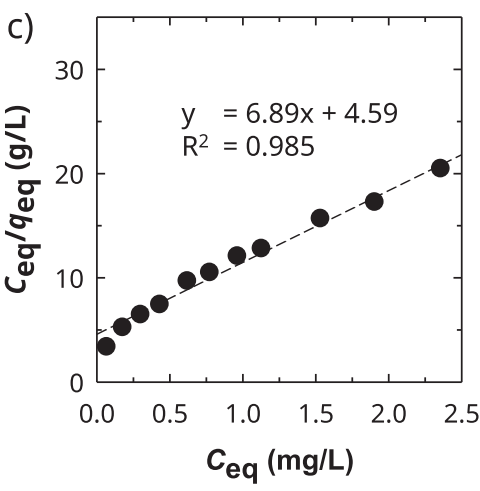

g)

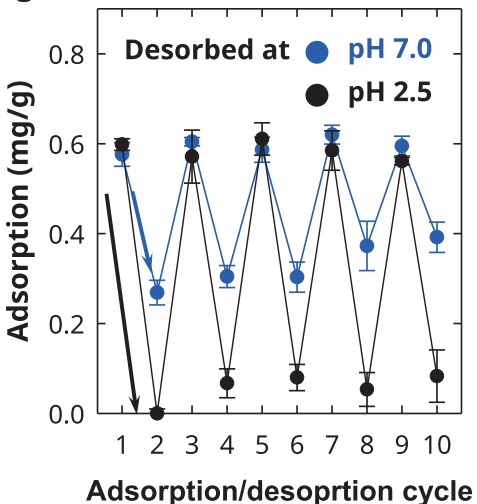

i)

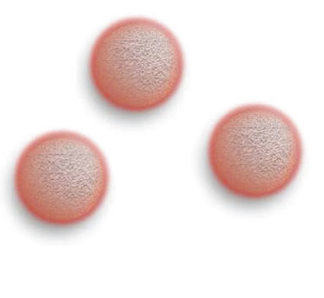

Fig. 6 Adsorption behavior of methylene blue (MB) on CNF-shelled microparticles. a Chemical structure of MB. b Equilibrium adsorption isotherm. c Langmuir isotherms of MB on microparticles at $23^{\circ} \mathrm{C}$, where the dotted lines are obtained by fitting the Langmuir model. d Demonstration of MB adsorption before (left), during (center), and after (right) filtration, where $100 \mathrm{mg}$ of CNF-shelled microparticles are retained in the $0.2 \mu \mathrm{m}$ PTFE membrane filter. e Light transmittance spectrum and $\mathbf{f}$ photograph of the MB solution before and after filtration. $\mathrm{g}$ Repeated adsorption/desorption behavior at $\mathrm{pH} 7.0$ and 2.5 through filtration. $\mathbf{h}$ Chemical structure of acridine orange hydrochloride. i Confocal laser scanning microscopy image of CNF-shelled microparticles, where surfaces are stained with acridine orange. Copyright 2019, The Royal Society of Chemistry and g). Similar experiments were conducted using other CNFs, such as microfibrillated cellulose, for the preparation of microparticles. However, the particles had a broader size distribution with low dispersibility in water. When water-soluble carboxymethyl cellulose was used, the resulting emulsion had poor structural stability, partially breaking down in the course of polymerization. Therefore, TEMPO-oxidized CNFs have been found to be suitable for preparing microparticles with good dispersibility and a narrow size distribution.
The molecular adsorption behavior of CNFs on the surfaces of particles was investigated using methylene blue (MB) as a cationic dye (Fig. 6a-c). At pH 7.0, the CNFs were shown to have a higher $\mathrm{MB}$ adsorption than at $\mathrm{pH} 2.5$. This was assumed to occur because the TEMPO-oxidized CNFs present on the particle surfaces had a large number of carboxylate groups: At $\mathrm{pH} 2.5$, the carboxylate groups on the surfaces of the CNFs could not form an ionic bond with $\mathrm{MB}$, and therefore, physical adsorption was thought to be the only adsorption mechanism at work. On the other hand, 
at $\mathrm{pH}$ 7.0, these groups could interact with MB through not only physical adsorption but also ionic bonding. Thus, the obtained CNF-shelled microparticle results indicated that the equilibrium adsorption isotherm was dependent on the $\mathrm{pH}$ conditions. Furthermore, the experimental data at $\mathrm{pH} 2.5$ were fitted with the Langmuir model, revealing good correspondence at low concentrations (Fig. 6c). In other words, our results suggested that theoretically estimated singlelayer adsorption occurred at low concentrations.

To take advantage of this adsorption characteristic, filtration was used to perform MB adsorption experiments (Fig. 6d). MB adsorption on the particle surfaces occurred very quickly: $\sim 99 \%$ of $\mathrm{MB}$ was adsorbed when the $\mathrm{MB}$ solution $\left(5 \mathrm{mg} \mathrm{L}^{-1}\right)$ was passed through at $\sim 100 \mathrm{~mL} \mathrm{~min}^{-1}$ and $\mathrm{pH} 7.0$ (Fig. 6e, f). Moreover, MB was washed with phosphate buffer $(\mathrm{pH} 2.5)$ once adsorbed and was almost entirely removed, which allowed us to repeatedly perform adsorption experiments (Fig. 6g).

To visualize this molecular adsorption behavior, a confocal laser microscopic observation was carried out. Acridine orange (Fig. 6h) was used as the fluorescence dye. Our observations revealed that acridine orange did not exist in the interior of the particles and was selectively adsorbed only on the surfaces of the particles (Fig. 6i). The acridine orange layer appeared thicker than the CNF layer observed in SEM. This was assumed to be due to the resolution capability of the confocal laser microscope being $\sim 200 \mathrm{~nm}$ and its scanning depth being $\sim 0.7 \mu \mathrm{m}$.

\section{Summary}

By taking advantage of the high potential of CNFs as an emulsifier, we successfully produced homogenous composite films of CNFs and a water-insoluble plastic. As these preparation processes do not require organic solvents or high-heat processing, they are considered to be highly environmentally friendly and could also be achieved at a lower cost than the generation of conventional CNF-based composites. We expect the $\mathrm{CNF} /$ polymer nanocomposite film to be utilized as a transparent material with excellent mechanical properties and low thermal expansion coefficients. Moreover, core-shell microparticles, whose shell and core are CNFs and a polymer, respectively, can be prepared from the Pickering emulsion. As the microparticles are covered with plant-based CNFs, they are expected to be utilized as functional microparticles. The biocompatibility of the CNF shells could open up new possibilities for biomedical or cosmetic applications. Moreover, the particles could be used for environmental applications by utilizing the carboxy groups of the CNFs.

Acknowledgements This research was supported by Grants-in-Aid for Scientific Research Grant numbers JP17K15298 and JP20K15567 from the Japan Society for the Promotion of Science (JSPS) and JSTMirai R\&D Program Grant number JPMJMI17ED from the Japan Science and Technology Agency (JST).

\section{Compliance with ethical standards}

Conflict of interest The authors declare that they have no conflict of interest.

Publisher's note Springer Nature remains neutral with regard to jurisdictional claims in published maps and institutional affiliations.

Open Access This article is licensed under a Creative Commons Attribution 4.0 International License, which permits use, sharing, adaptation, distribution and reproduction in any medium or format, as long as you give appropriate credit to the original author(s) and the source, provide a link to the Creative Commons license, and indicate if changes were made. The images or other third party material in this article are included in the article's Creative Commons license, unless indicated otherwise in a credit line to the material. If material is not included in the article's Creative Commons license and your intended use is not permitted by statutory regulation or exceeds the permitted use, you will need to obtain permission directly from the copyright holder. To view a copy of this license, visit http://creativecommons. org/licenses/by/4.0/.

\section{References}

1. Wu X, Moon RJ, Martini A. Tensile strength of I $\beta$ crystalline cellulose predicted by molecular dynamics simulation. Cellulose. 2014;21:2233-45

2. Saito T, Kuramae R, Wohlert J, Berglund LA, Isogai A. An ultrastrong nanofibrillar biomaterial: the strength of single cellulose nanofibrils revealed via sonication-induced fragmentation. Biomacromolecules. 2013;14:248-53.

3. Sakurada I, Nukushina Y, Ito T. Experimental determination of the elastic modulus of crystalline regions in oriented polymers. J Polym Sci. 1962;57:651-60.

4. Šturcová A, Davies GR, Eichhorn SJ. Elastic modulus and stresstransfer properties of tunicate cellulose whiskers. Biomacromolecules. 2005;6:1055-61.

5. Iwamoto S, Kai W, Isogai A, Iwata T. Elastic modulus of single cellulose microfibrils from tunicate measured by atomic force microscopy. Biomacromolecules. 2009;10:2571-6.

6. Hori R, Wada M. The thermal expansion of wood cellulose crystals. Cellulose. 2005;12:479-84.

7. Diaz JA, Wu X, Martini A, Youngblood JP, Moon RJ. Thermal expansion of self-organized and shear-oriented cellulose nanocrystal films. Biomacromolecules. 2013;14:2900-8.

8. Azizi Samir MAS, Alloin F, Dufresne A. Review of recent research into cellulosic whiskers, their properties and their application in nanocomposite field. Biomacromolecules. 2005;6:612-26.

9. Eichhorn SJ, Dufresne A, Aranguren M, Marcovich NE, Capadona JR, Rowan SJ, et al. Review: Current international research into cellulose nanofibres and nanocomposites. J Mater Sci. 2010;45:1-33.

10. Siró I, Plackett D. Microfibrillated cellulose and new nanocomposite materials: a review. Cellulose. 2010;17:459-94.

11. Saito T, Nishiyama Y, Putaux JL, Vignon M, Isogai A. Homogeneous suspensions of individualized microfibrils from TEMPOcatalyzed oxidation of native cellulose. Biomacromolecules. 2006;7:1687-91. 
12. Isogai A, Saito T, Fukuzumi H. TEMPO-oxidized cellulose nanofibers. Nanoscale. 2011;3:71-85.

13. Okita Y, Fujisawa S, Saito T, Isogai A. TEMPO-oxidized cellulose nanofibrils dispersed in organic solvents. Biomacromolecules. 2011;12:518-22.

14. Fujisawa S, Saito T, Kimura S, Iwata T, Isogai A. Surface engineering of ultrafine cellulose nanofibrils toward polymer nanocomposite materials. Biomacromolecules. 2013;14:1541-6.

15. Shimizu M, Fukuzumi H, Saito T, Isogai A. Preparation and characterization of TEMPO-oxidized cellulose nanofibrils with ammonium carboxylate groups. Int J Biol Macromol. 2013;59:99-104.

16. Fujisawa S, Ikeuchi T, Takeuchi M, Saito T, Isogai A. Superior reinforcement effect of TEMPO-oxidized cellulose nanofibrils in polystyrene matrix: optical, thermal, and mechanical studies. Biomacromolecules 2012;13:2188-94.

17. Soeta H, Fujisawa S, Saito T, Berglund L, Isogai A. Lowbirefringent and highly tough nanocellulose-reinforced cellulose triacetate. ACS Appl Mater Interfaces. 2015;7:11041-6.

18. Kalashnikova I, Bizot H, Cathala B, Capron I. New pickering emulsions stabilized by bacterial cellulose nanocrystals. Langmuir. 2011;27:7471-9.

19. Fujisawa S, Togawa E, Kuroda K. Nanocellulose-stabilized Pickering emulsions and their applications. Sci Technol Adv Mater. 2017;18:959-71.

20. Salas C, Nypelö T, Rodriguez-Abreu C, Carrillo C, Rojas OJ. Nanocellulose properties and applications in colloids and interfaces. Curr Opin Colloid Interface Sci. 2014;19:383-96.

21. Fujisawa S, Togawa E, Kuroda K. Facile route to transparent, strong, and thermally stable nanocellulose/polymer nanocomposites from an aqueous pickering emulsion. Biomacromolecules. 2017;18:266-71.

22. Biswas SK, Sano H, Shams MI, Yano H. Three-dimensionalmoldable nanofiber-reinforced transparent composites with a hierarchically self-assembled "reverse" nacre-like architecture. ACS Appl Mater Interfaces. 2017;9:30177-84.
23. Biswas SK, Tanpichai S, Witayakran S, Yang X, Shams MI, Yano $\mathrm{H}$. Thermally superstable cellulosic-nanorod-reinforced transparent substrates featuring microscale surface patterns. ACS Nano. 2019;13:2015-23.

24. Yan Y, Herzele S, Mahendran AR, Edler M, Griesser T, Saake B, et al. Microfibrillated lignocellulose enables the suspensionpolymerisation of unsaturated polyester resin for novel composite applications. Polym. 2016;8:255.

25. Shams MI, Yano H. Doubly curved nanofiber-reinforced optically transparent composites. Sci Rep. 2015;5:16421.

26. Noguchi S, Sato K, Yamamoto K, Kadokawa J-I. Preparation of composite and hollow particles from self-assembled chitin nanofibers by Pickering emulsion polymerization. Int J Biol Macromol. 2019;126:187-92.

27. Noguchi S, Yamamoto K, Kadokawa J-I. Preparation of chitinbased fluorescent hollow particles by Pickering emulsion polymerization using functional chitin nanofibers. Int J Biol Macromol. 2019;157:680-6.

28. Fujisawa $\mathrm{S}$, Togawa E, Kuroda K, Saito T, Isogai A. Fabrication of ultrathin nanocellulose shells on tough microparticles: via an emulsion-templated colloidal assembly: towards versatile carrier materials. Nanoscale. 2019;11:15004-9.

29. Lok KP, Ober CK. Particle size control in dispersion polymerization of polystyrene. Can J Chem. 1985;63:209-16.

30. Tseng CM, Lu YY, El-Aasser MS, Vanderhoff JW. Uniform polymer particles by dispersion polymerization in alcohol. J Polym Sci Part A Polym Chem. 1986;24:2995-3007.

31. Ober CK, Lok KP, Hair ML. Monodispersed, micron-sized polystyrene particles by dispersion polymerization. J Polym Sci Polym Lett Ed. 1985;23:103-8.

32. Goi Y, Fujisawa S, Saito T, Yamane K, Kuroda K, Isogai A. Dual functions of tempo-oxidized cellulose nanofibers in oil-in-water emulsions: a pickering emulsifier and a unique dispersion stabilizer. Langmuir. 2019;35:10920-6. 